\title{
Urothelial Carcinoma: Optimal Treatment Options in First-Line and Beyond
}

\author{
Ursula Vog $\left.\right|^{1 *}$
}

\section{ABSTRACT}

For many years, the standard treatment approach in first-line for advanced urothelial carcinoma (UC) was platinum-based chemotherapy, with the choice of agent dependent on cisplatin-eligibility and relatively limited second-line therapy options included taxanes or vinflunine, thus although with missing approval in Switzerland. ${ }^{1}$ In the last years, the treatment landscape has changed due to the emergence of new targeted approaches, including immune checkpoint inhibitors (ICIs). ${ }^{2}$ In Switzerland, programmed death-protein 1 (PD-1)/programmed death-ligand 1 (PD-L1) inhibitors including pembrolizumab, atezolizumab and nivolumab are currently approved for the treatment of locally advanced or metastatic UC in adults having progressed on platinum-based chemotherapy $^{3-5}$, as well as pembrolizumab and atezolizumab in PD-L1 positive treatment-naïve patients if platinum unfit. Evidence from a recent phase III trial has led to a new standard of care with a switch maintenance approach with the PD-L1 inhibitor avelumab for UC patients who are progression-free following platinum-based chemotherapy. Several novel emerging targeted therapies such as antibody-drug conjugates (ADC) or fibroblast growth factor receptor (FGFR) inhibitors are also being investigated in treatmentnaïve as well as pretreated, platinum-and checkpoint inhibitor refractory patients with locally advanced or metastatic UC. This article provides an overview of current treatment options in first- and subsequent-line settings.

Keywords: immune checkpoint inhibitors (ICIS), programmed death-protein 1 (PD-1) inhibitors, programmed death-ligand 1 (PD-L1) inhibitors, urothelial carcinoma (UC)

\section{INTRODUCTION}

FIRST-LINE THERAPY OPTIONS: A NEW STANDARD APPROACH

A few randomized phase II/III trials explored the role of immunotherapy for urothelial carcinoma (UC) in the first-line setting, either as monotherapy and/or in combination with chemotherapy. ${ }^{6-10}$ The open-label, phase III JAVELIN Bladder-100 study was conceived to prove the benefit of a maintenance therapy with a programmed death-ligand 1 (PD-L1) inhibitor after initial response to 4 to 6 cycles of platinum-based chemotherapy, demonstrating that maintenance with avelumab in addition to best supportive care (BSC) significantly prolonged overall survival (OS) versus BSC alone (median OS, 21.4 months vs 14.3 months; HR: 0.69 [95\% Cl: 0.56-0.86]; $\mathrm{p}=0.001){ }^{7}$ A post hoc analysis of the trial recently assessed the efficacy of avelumab maintenance plus BSC versus BSC alone by the duration of treatment-free interval (TFI), defined as the time from completion of chemotherapy to start of first-line maintenance. Results showed that OS and PFS benefits were consistent across all TFI subgroups, including TFI: $4-<6$ weeks, TFI: $6-<8$ weeks, TFI: $8-10$ weeks. ${ }^{11}$ The trial also provides quality of life data supporting the benefit of avelumab maintenance without deterioration of the overall health status. ${ }^{12}$

Results of IMvigor-130 and KEYNOTE-361 have failed to support the combinational use of first-line platinum-based chemotherapy and a programmed death-protein 1 (PD-1) or PD-L1 inhibitor as the frontline treatment for advanced or metastatic UC. ${ }^{8,9}$ Both trials compared atezolizumab and pembrolizumab in combination with platinum-based chemotherapy. In addition, the third arm in both trials enrolled patients to receive first-line immune checkpoint inhibitors (ICI) monotherapy compared to platinum-based chemotherapy alone, including a retrospective subgroup analysis on PD-L1 expression status. Progression-free survival (PFS) and OS were analyzed as pre-specified co-primary endpoints in both trials. Overall, the final analysis of the two trials showed a modest improvement in PFS in patients receiving combined chemotherapy and atezolizumab in IMvigor-130 (HR: 0.82 [95\% CI: 0.70-0.96]; $\mathrm{p}=0.007$ ) or pembrolizumab in KEYNOTE-361 (HR: 0.78 [95\% CI: 0.65-0.93]; $\mathrm{p}=0.0033$ ), as compared with chemotherapy alone. Results from both trials further indicated

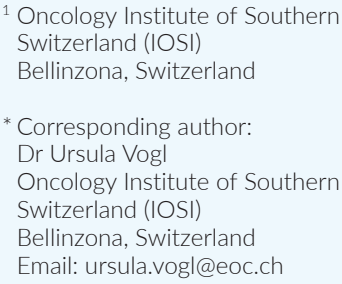

DOI: 10.36000/hbT.OH.2021.09.046 ISSN: 2673-2092 (Print) and 2673 2106 (Online)

\section{This article was received on} September 10, 2021.

This article was accepted on October 04, 2021.

This article was published on October 15, 2021.

Vogl U. Urothelial Carcinoma: Optimal Treatment Options in First-Line and Beyond. healthbook TIMES Onco Hema. 2021;(9):32-37. 


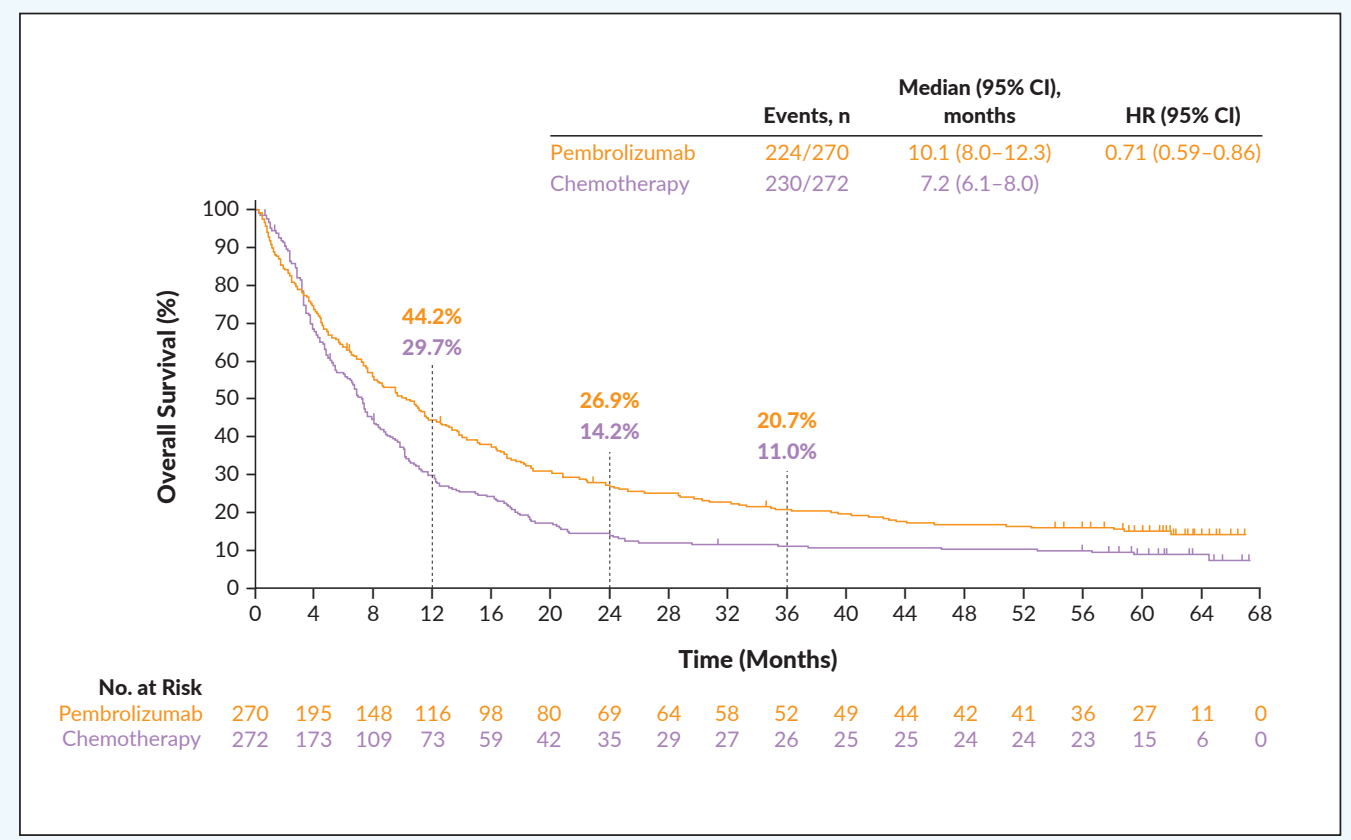

Figure 1. Second-line pembrolizumab versus platinum-based chemotherapy leads to improved overall survival in pretreated patients with advanced urothelial carcinoma. Adapted from Bellmunt et al. 2021. ${ }^{17}$ that the addition of ICI to platinum-based chemotherapy does not significantly improve $O S$ in the first-line setting. Data from IMvigor-130, KEYNOTE-361 and DANUBE also demonstrated that although the duration of response (DoR) seen after first-line treatment with ICIs tends to be longer than with chemotherapy, only a small proportion of patients (i.e., those with high PD-1/PD-L1 expression status) experience this benefit. ${ }^{8-10}$ Notably, the initial response after a few months of treatment is superior with chemotherapy (CT) for most UC patients.

Secondary/exploratory endpoint data from IMvigor- $130^{13}$ and KEYNOTE-36 $1{ }^{14}$ provide supportive evidence for the current EMA label for atezolizumab and pembrolizumab in platinineligible patients with high PD-L1 expression. In a subgroup of cisplatin-ineligible patients with high PD-L1 status $(\geq 5 \%)$ receiving the first-line atezolizumab in IMvigor-130, the investigator-assessed overall response rate (ORR) was 38\% in atezolizumab-treated patients versus $33 \%$ in platinum/ gemcitabine-treated patients. ${ }^{13}$

\section{EMERGING SECOND- AND SUBSEQUENT-LINE OPTIONS}

Pembrolizumab is currently the only ICI that demonstrated a significant OS benefit as a second-line treatment in a phase III trial. ${ }^{15,16}$ After 3 years of treatment follow-up, the KEYNOTE-045 trial demonstrated an improvement in OS with pembrolizumab compared with chemotherapy in UC patients who progressed after platinum-based chemotherapy. ${ }^{15}$ Median OS with pembrolizumab was 10.1 versus 7.2 months with chemotherapy (HR: 0.72 [95\% CI: 0.59-0.87]; $\mathrm{p}=0.0003)$. In the recently presented data at ASCO 2021, after 5 years of follow-up, pembrolizumab versus chemotherapy continued to improve the median OS (10.1 months vs 7.2 months) (Figure 1), as well as ORR (21.9\% vs $11.0 \%)$ and the median DoR (29.7 months vs 4.4 months) in this patients population. ${ }^{17}$ In contrast, in the hierarchically tested and pre-specified PD-L1 positive/first endpoint populations, atezolizumab was not associated with significantly longer OS versus chemotherapy in patients with platinum-refractory metastatic UC in the phase III IMvigor 211 trial. ${ }^{16}$ However, the safety profile for atezolizumab was favorable compared with chemotherapy in this patient setting.

Treatment after progression on platinum-based chemotherapy and ICI is challenging due to limited treatment options. New therapeutic agents are therefore warranted. Erdafitinib is a novel fibroblast growth factor receptor (FGFR) kinase inhibitor used to treat advanced UC patients with susceptible $F G F R 3$ or FGFR2 genetic alterations. In an international open-label phase II trial, 99 patients with FGFR-altered UC were treated with an optimized dosing schedule of once-daily erdafitinib $(8 \mathrm{mg}) \cdot{ }^{18}$ All study participants had progressed after or during $\geq 1$ line of prior platinum-based CT; notably, prior treatment with a prior ICI was allowed. The primary endpoint of ORR was $40 \%$, with $3 \%$ of patients achieving a complete response and $37 \%$ a partial response. ${ }^{19}$ Stable disease (SD) occurred in $39 \%$ of patients. These data show that erdafitinib provides unprecedented response rates, even in patients with visceral metastases. After a median follow-up of 24 months, the overall median PFS and OS for all patients were 5.52 months and 11.3 months, respectively. ${ }^{20}$ Although most patients progressed by 18 months, such an additional survival benefit is valuable in the third-line setting. While erdafitinib is a convenient oral medicine to administer with a promising clinical activity, this agent was associated with any-grade adverse events (AEs) such as hyperphosphatemia $(72 \%)$ as well as skin, nail and ocular-related events (all up to $50 \%) .{ }^{18,19}$ Encouraging data in combination with a checkpoint inhibitor in cisplatin-unfit patients has recently been presented from a phase Ib study by Powles and colleagues at the 2021 ESMO meeting and warrants further investigation in future trials. ${ }^{21}$ 


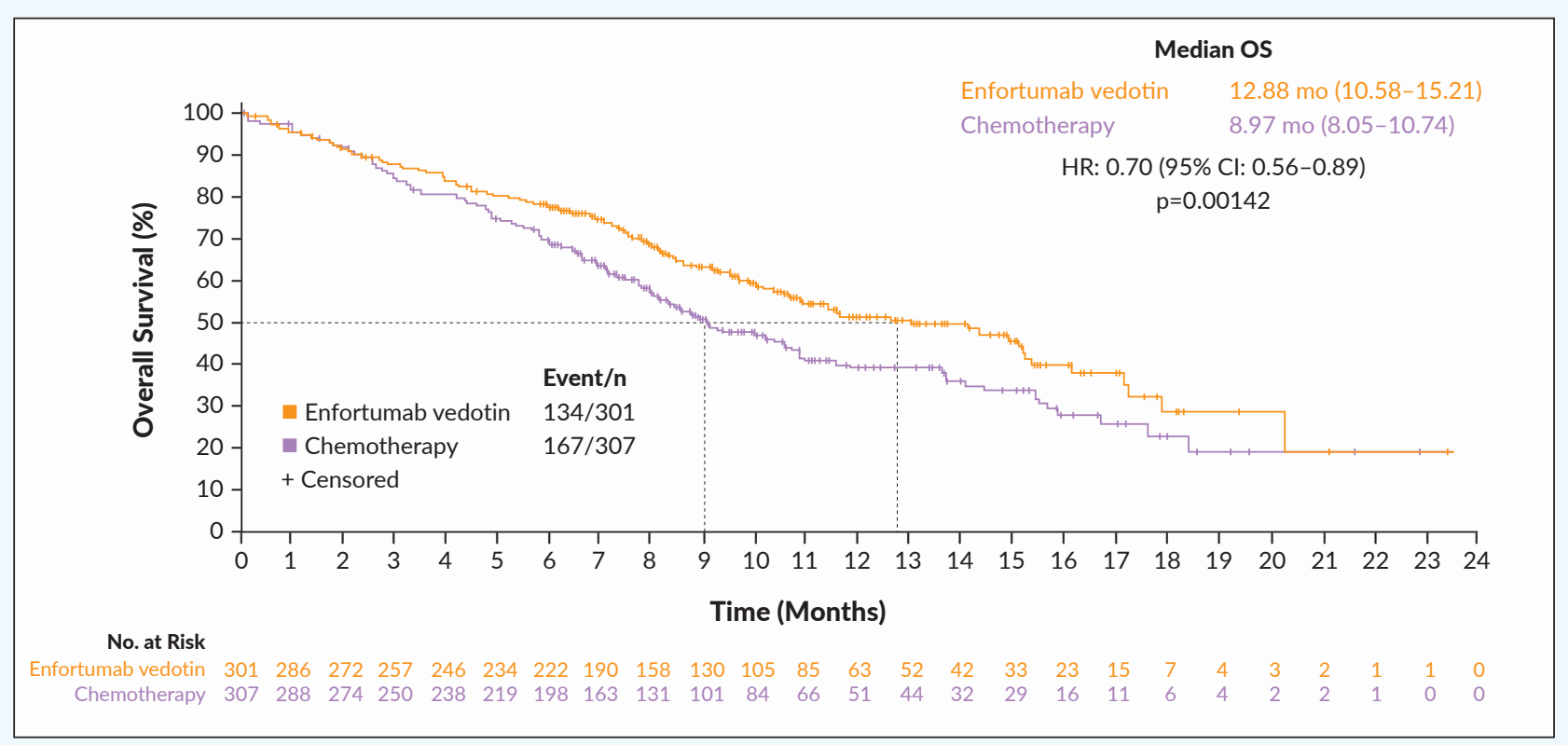

Figure 2. Overall survival (OS) in phase III EV-301 trial. Adapted from Powles et al. 2021.22

Enfortumab vedotin, an antibody-drug conjugate (ADC) comprised of a fully human monoclonal antibody and the microtubule-disrupting agent monomethyl auristatin $\mathrm{E}$, is another emerging new UC treatment being investigated in clinical trials in the third-line setting. This agent targets nectin-4, which is highly expressed in UC. The open-label, phase II EV-301 trial evaluated enfortumab vedotin in patients who had progressed following PD-1/PD-L1 therapy and who had received a prior platinum-containing regimen. ${ }^{22}$ The primary endpoint was OS, which was improved following treatment with enfortumab vedotin versus chemotherapy (12.88 months vs 8.97 months; HR: 0.70 [95\% CI: 0.56-0.89]; $\mathrm{p}=0.00142$ ) (Figure 2). Notably, treatment can be held and restarted in case if AEs of special interest, such as neuropathy, skin reactions or hyperglycemia, are experienced. However, enfortumab vedotin is generally well-tolerated, with most treatment-relevant AEs of special interest reported as mild or moderate. In the single-arm 2-cohort, EV-201 study, enrolled platinum-naïve, cisplatin-ineligible patients with prior anti-PD-1/L1 treatment with locally advanced or metastatic urothelial carcinoma. The first previously presented analysis results of EV-201 Cohort 2 showed an ORR of $52 \%$ with a CR rate of $20 \%$ and a median DoR of 10.9 months. ${ }^{23}$ The updated results with an additional 3 months follow-up were consistent with those of the primary analysis with an ORR of $51 \%$, CR of $22 \%$ and a median DoR of 13.8 months. ${ }^{24}$ Based on the results of phase II EV-301 trial, the FDA recently granted regular approval to enfortumab vedotin for patients with locally advanced or metastatic UC, who have received a PD-1 or PD-L1 inhibitor and platinum-containing chemotherapy or are ineligible for cisplatin-containing chemotherapy and have previously received $\geq 1$ prior lines of therapy in the neoadjuvant/adjuvant, locally advanced or metastatic setting.

\section{CURRENT TREATMENT LANDSCAPE IN SWITZERLAND}

PD-1/PD-L1 inhibitors have redefined the standard of care for bladder cancer in different ways and treatment lines (Figure 3). Three of these agents, atezolizumab, nivolumab and pembrolizumab, are approved in Switzerland for the treatment of adult patients with locally advanced or metastatic UC who have received prior platinum-containing chemotherapy of those pembrolizumab has the highest evidence from a randomized phase III trial. ${ }^{3-5}$ Recently, avelumab was approved by $\mathrm{EMA}^{25}$ and Swissmedic ${ }^{26}$ as monotherapy for first-line maintenance therapy in patients with locally advanced or metastatic UC whose disease has not progressed on first-line platinum-based induction chemotherapy. First-line atezolizumab and pembrolizumab are also approved in advanced UC patients who are considered cisplatin-ineligible and whose tumors have high PD-L1 expression, although with low evidence from singlearm phase II trials. ${ }^{17}$ In Switzerland, pembrolizumab is currently the only ICI approved in advanced UC patients with PD-L1-positive tumors and who are cisplatinineligible. $^{27}$ Erdafitinib for patients with FGFR2,3 or some other alterations and enfortumab vedotin are not yet approved in Europe or Switzerland but have already entered guideline recommendations due to positive phase II and III trials. In Switzerland, erdafitinib and enfortumab vedotin can be offered to patients through a compassionate use program.

\section{FUTURE PERSPECTIVES}

Adjuvant treatment strategies for muscle-invasive bladder cancer have been limited to those being Cisplatinum-fit and have not received neoadjuvant treatment. For those not being able to receive cisplatin, no specific adjuvant treatment is recommended. The beneficial role of PD-1 and PD-L1 inhibitors in advanced or metastatic UC has given rationale for conceptualizing clinical trials with these drugs also in the adjuvant setting. Although IMvigor-010 with atezolizumab versus placebo observation did not meet its primary endpoint, data from an exploratory analysis showed that patients with muscle-invasive bladder cancer (MIBC) who had detectable circulating tumor DNA (ctDNA) were more likely to benefit from treatment with adjuvant atezolizumab monotherapy, compared with those without ctDNA negative ones (median 


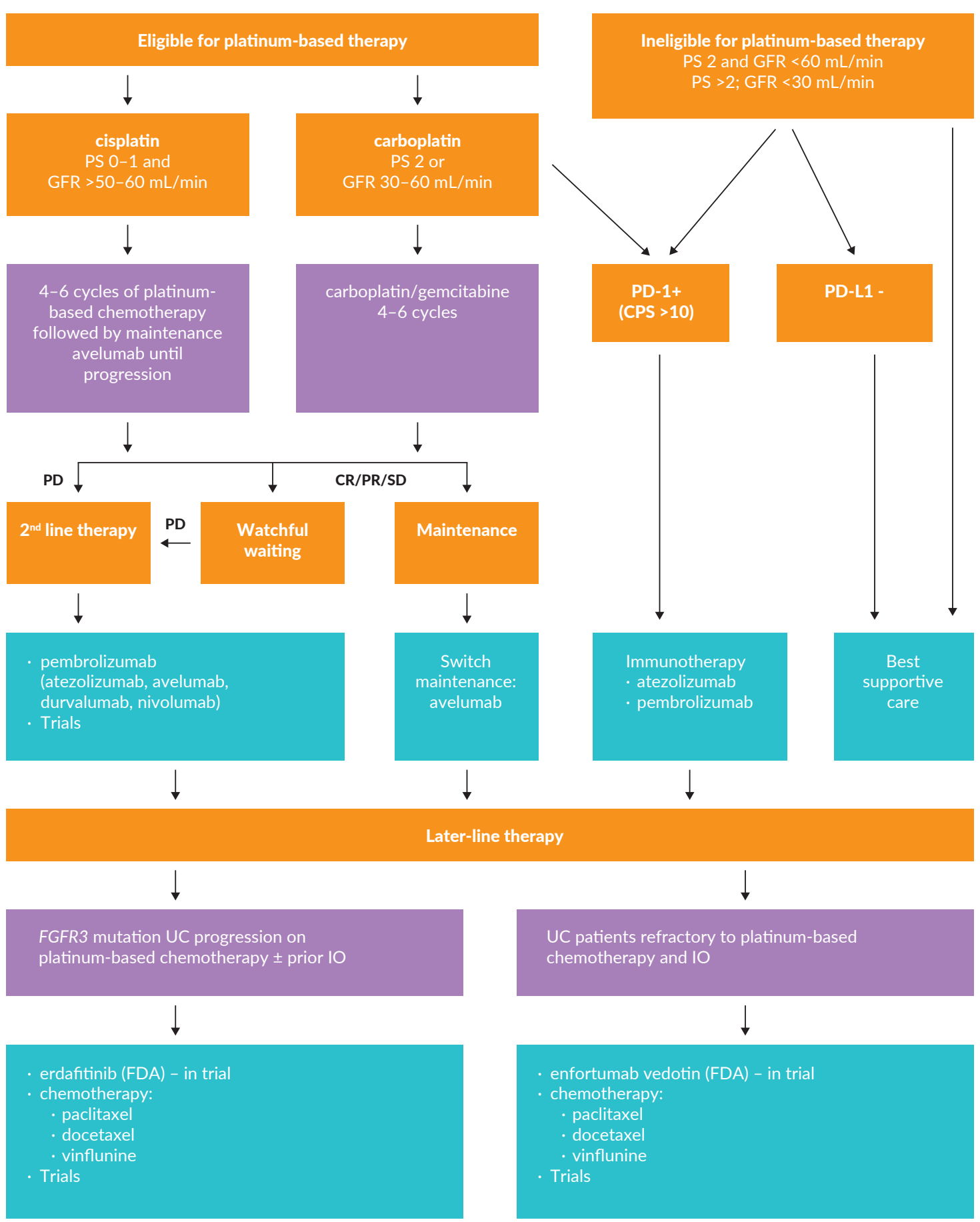

Figure 3. Current treatment paradigm in advanced urothelial carcinoma. Enfortumab Vedotin (EV), erdafitinib, durvalumab, taxanes and vinflunine are not approved in Switzerland. BSC, best supportive care; CR, complete response; DD-MVAC, dose-dense methotrexate vinblastine doxorubicin cisplatin; EV, enfortumab vedotin; FDA, Food and Drug Administration; FGFR, pan-fibroblast growth factor receptor tyrosine kinase inhibitor; GFR, glomerular filtration rate; IO, immunotherapy; PD-L1, programmed cell death 1; PR, partial response; PD, progressive disease; PS, performance status; SD, stable disease; UC, urothelial carcinoma. Adapted from the European Association of Urology (EAU) Guidelines March 2021 for metastatic urothelial cancer. ${ }^{28}$

disease-free survival [DFS], 5.9 months vs 4.4 months).${ }^{29}$ At an interim analysis, $O S$ was superior favoring atezolizumab versus observation in the ctDNA-positive population (median, 25.8 months vs 15.8 months; HR: 0.59 [95\% CI: 0.41-0.86]). These data further improve our understanding of the disease and will inform a new phase III study in patients with ctDNApositive MIBC.
In the double-blind CheckMate-274 trial, nivolumab treatment for up to 12 months following radical surgery in patients with high-risk MIBC resulted in a statistically significant and clinically meaningful improvement in the first endpoint DFS in this setting (21.0 months vs 10.9 months with placebo; HR: 0.70 [95\% CI: $0.54-0.89$ ]; $\mathrm{p}<0.001) .{ }^{30} \mathrm{DFS}$ in the subgroup of patients with PD-L1-positive tumors was also improved with 


\section{healthbook TIMES Oncology Hematology}

nivolumab; however, the median was not yet reached by the time of analysis. In terms of secondary endpoint, non-urothelial tract recurrence-free survival (NUTRFS) was also improved with nivolumab versus placebo in both intention-to-treat (ITT) population (24.6 months vs 13.7 months; HR: 0.72 [95\% CI: $0.58-0.89]$ ) and in patients with PD-L $\geq 1 \%$ (not reached vs 10.9 months with placebo; HR: 0.54 [95\% CI: 0.38-0.77]). Finally, OS data are still immature and most likely have to be awaited to announce before adjuvant nivolumab would become a truly practice-changing standard of care. Furthermore, the results from the ongoing AMBASSADOR Alliance trial will help broaden our understanding of the role of adjuvant immunotherapy, in particular, how pembrolizumab given postcystectomy might improve survival outcomes in patients with high-risk MIBC. ${ }^{31}$

Recent trials showing a pathological response with immunotherapy in the neoadjuvant setting include the phase II ABACUS ${ }^{32}$ and PURE-0 $1^{33}$ studies, which demonstrated promising results with atezolizumab and pembrolizumab in pre-surgical UC patients. Combination regimens of chemotherapy and immunotherapy agents in the neoadjuvant setting are also being evaluated in phase II clinical trials (e.g., BLASST- $\left.1^{34}\right)$.

For the time being, classic neoadjuvant chemotherapy will remain the standard of care for patients for MIBC patients from T2 muscle-invasive disease who are cisplatin-fit. The VESPER phase III trial recently presented at the ESMO meeting showed superiority in terms of metastatic PFS at
3 years and OS and an impressive pathological complete response ( $\mathrm{pCR}$ ) rate of 6 cycles of dose-dense methotrexate, vinblastine, doxorubicin and cisplatin (MVAC). ${ }^{35}$ Therefore, dose dense MVAC should be considered the regimen of choice in the neoadjuvant setting for those patients fit enough to receive this potentially more effective, thus toxic treatment.

\section{CONCLUSIONS}

- Based on recent practice-changing trials, platinum-based chemotherapy followed by an immune checkpoint inhibitor (ICI) as a switch maintenance concept, in patients not progressing on chemotherapy, responding or stable disease has become the current gold standard of care for patients with advanced or metastatic urothelial cancer (UC).

- First-line ICI such as pembrolizumab or atezolizumab is an option for cisplatin-ineligible patients who have high PD-L1 expression, while second-line ICI immunotherapy offers an additional option for patients progressing during or after initial platinum-based chemotherapy.

- Several factors such as FGFR mutational status, comorbidities and adverse events should be established taken into consideration for the decision of third-line treatment of advanced UC patients with erdafitinib or enfortumab vedotin. These agents, although are not yet approved in Switzerland, but available through a compassionate use program.

- The use of ctDNA and other biomarkers will provide further insights and enable a more individualized treatment approach, provided large ongoing prospective trials will confirm their utility.

\section{CONFLICT OF INTEREST}

The author declares no conflict of interest.

REFERENCES

1. Bladder Cancer: ESMO Clinical Practice Guideline ESMO 2019. [Accessed June 2021]. Available from: www. esmo.org/guidelines/genitourinary-cancers/bladder-cancer. 2. Bellmunt J, Powles T, Vogelzang NJ. A review on the evolution of PD-1/PD-L1 immunotherapy for bladder cancer: The future is now. Cancer Treat Rev. 2017:54:58-67. cer: The future is now. Cance
doi:10.1016/j.ctrv.2017.01.007

doi:10.1016/j.ctrv.2017.01.007
3. TECENTRIQ (atezolizumab). Product information. Swissmedic 2019. [Accessed June 2021]. Available from: www. swissmedicinfo.ch.

4. KEYTRUDA (pembrolizumab). Product information. Swissmedic 2020. [Accessed June 2021]. Available from: www. swissmedicinfo.ch.

5. OPDIVO (nivolumab). Product information. Swissmedic 2020. [Accessed June 2021]. Available from: www.swissmedicinfo.ch.

6. Galsky MD, Mortazavi A, Milowsky MI, et al. Randomized Double-Blind Phase II Study of Maintenance Pembrolizumab Versus Placebo After First-Line Chemotherap in Patients With Metastatic Urothelial Cancer. J Clin Oncol. 2020;38(16):1797-1806. doi:10.1200/JCO.19.03091

7. Powles T, Park SH, Voog E, et al. Avelumab Maintenance Therapy for Advanced or Metastatic Urothelial Carcinoma. N Engl J Med. 2020;383(13):1218-1230. doi:10.1056/NEJMoa2002788

8. Galsky MD, Arija JÁA, Bamias A, et al. Atezolizumab with or without chemotherapy in metastatic urothelial can- cer (IMvigor 130): a multicentre, randomised, placebo-controlled phase 3 trial. Lancet. 2020;395(10236):1547-1557. doi:10.1016/S0140-6736(20)30230-0

9. Alva A, Csőszi T, Ozguroglu M, et al. LBA23 Pembrolizuma (P) combined with che (C) vs $\mathrm{C}$ alone mab (P) combined with chemotherapy (C) vs $\mathrm{C}$ alone as firsline (1L) therapy for advanced urothelial carcinoma (UC): KEYNOTE-361. Ann

annonc.2020.08.2252
10. Powles T, van der Heijden MS, Castellano D, et al. Durvalumab alone and durvalumab plus tremelimumab versus chemotherapy in previously untreated patients with unresectable, locally advanced or metastatic urothelial carcinom (DANUBE): a randomised, open-label, multicentre, phase 3 trial. Lancet Oncol. 2020;21(12):1574-1588. doi:10.1016 S1470-2045(20)30541-6

11. Sridhar SS, Powles T, Loriot Y, et al. Avelumab first-line (IL) maintenance for advanced urothelial carcinom (UC) in the JAVELIN Bladder 100 trial: Subgroup analysis by duration of treatment-free interval (TFI) from end of chemotherapy to start of maintenance. $J$ Clin Oncol. 2021;39(15_suppl):4527-4527. doi:10.1200/ JCO.2021.39.15 suppl.4527

12. Powles TB, Kopyltsov E, Su P et al. Patient-reported outcomes (PROs) from JAVELIN Bladder 100: Avelumab firstline (1L) maintenance + best supportive care (BSC) vs BSC lone for anced urot lial arinom (UC). Poster presentation presented at: ESMO Virtual Congress 2020; 19-21
September 2020

13. Galsky MD, Bamias A, Arranz Arija JA, et al. Atezolizumab (atezo) monotherapy versus chemotherapy in previously untreated locelly advanced or metastatic urothelial viously untreated locally advanced or metastatic urothelial (clinical outcomes by PD-L1 status in cisplatin (cis)-ineligible pts from the phase III IMvigor130 study.J Clin Oncol. 2021;39(6_suppl):434-434. doi:10.1200/ JCO.2021.39.6_suppl.434

14. Powles T, Csőszi T, Ozguroglu M, et al. 1L pembrolizumab (pembro) versus chemotherapy (chemo) for choice-ofcarboplatin patients with advanced urothelial carcinoma (UC) in KEYNOTE-361.JClin Oncol.2021;39(6_suppl):450-450. doi:10.1200/JCO.2021.39.6_suppl.450

15. Necchi A, Fradet Y, Bellmunt J, et al. Three-year followup from the phase III KEYNOTE-045 trial: Pembrolizumab (Peror the (Pembro) versus investigator's choice (paclitaxel, docetaxel, or vinflunine) in recurrent, advanced urothelial cancer (UC). Ann Oncol. 2019;30:v366-v367. doi:10.1093/annonc/ mdz249.018

16. Powles T, Durán I, van der Heijden MS, et al. Atezolizumab versus chemotherapy in patients with platinum-treated locally advanced or metastatic urothelial carcinoma (IMvigor211): a multicentre, open-label, phase 3 randomised controlled trial. Lancet. 2018;391(10122):748-757. doi:10.1016/ S0140-6736(17)33297-X

17. Bellmunt J, Necchi A, De Wit R, et al. Pembrolizumab (pembro) versus investigator's choice of paclitaxel, docetaxel, 
Treatment Options in Urothelial Carcinoma

or vinflunine in recurrent, advanced urothelial cancer (UC): 5 -year follow-up from the phase 3 KEYNOTE-045 trial. $J$ Clin Oncol. 2021;39(15_suppl):4532-4532. doi:10.1200/ JCO.2021.39.15_suppl.4532

18. Siefker-Radtke AO, Necchi A, Park SH, et al. First results from the primary analysis population of the phase 2 study of

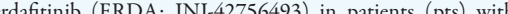
(pts) with ( $\mathrm{mUC})$ and FGFR alterations (FGFRalt). J Clin Oncol. 2018:36(15
suppl):4503-4503.

suppl.4503

19. Loriot Y, Necchi A, Park SH, et al. Erdafitinib in Locally Advanced or Metastatic Urothelial Carcinoma. N EnglJ Med. 2019;381(4):338-348. doi:10.1056/NEJMoa1817323

20. Siefker-Radtke A, Necchi A, Park S, GarcĀa-Donas J, Huddart R and Burgess E. ERDAFITINIB in locally advanced or metastatic urothelial carcinoma (mUC): Long-term outcomes in BLC2001. Abstract presented at: ASCO 2020 Virtual. Abstract 5015 .

21. Powles TB, Chistyakov V, Beliakouski V et al. Erdafitinib (ERDA) or ERDA plus cetrelimab (CET) for patients with (ERDA) or ERDA plus cetrelimab (CET) for patients with
metastatic or locally advanced urothelial carcinoma (mUC) metastatic or locally advanced urothelial carcinoma (mUC)
and Fibroblast Growth Factor Receptor alterations (FGFRa): First phase $(\mathrm{Ph})$ II results from the NORSE study. Oral presentation presented at: ESMO Congress 2021; 16-21 September 2021.

22. Powles T, Rosenberg JE, Sonpavde G, et al. Primary results of EV-301: A phase III trial of enfortumab vedotin versus cheotherapy in patients with previously treated locally advanced or metastatic urothelial carcinoma. J Clin Oncol. 2021:39(6 suppl):393-393. doi:10.1200/JCO.2021.39.6_suppl.393 23. Powles T, Rosenberg JE, Sonparde GP, et al. Enfortumab Vedotin in Previously Treated Advanced Urothelial Carcinoma. N Engl J Med. 2021;384(12):1125-1135. doi:10.1056/ NEJMoa2035807
24. McGregor BA et al. Enfortumab vedotin in cisplatin-ineligible patients with locally advanced or metastatic urothelia cancer who received prior PD-1/PD-L1 inhibitors: An updated analysis of EV-201 Cohort 2. Poster presentation presented at: 2021 ASCO Virtual Annual Meeting; 4-8 June 2021. Poster presentation 4524.

25. European Commission Approves BAVENCIO (ave-

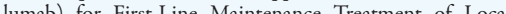
Advaced or Metratic Urotelial Carcino Adval June 2021]. Avalable from: www.pfizercom/new cessed June 2021]. Available from. Www.plizer.com/news/ press-release/press-release-detail/european-commissionapproves-bavencior-avelumab-first-line\#: : text=BAVENCIO\%C2\%AE\%20(avelumab)\%20is\%20indicated,\%2Dline $\% 20$ platinum $\% 2$ Dcontaining\%20chemotherapy.

26. $\mathrm{BAVENCIO}^{\circ}$ (avelumab). Product information. Swissme dic 2021. [Accessed August 2021]. Available from: https:// www.swissmedicinfo.ch.

27. Swiss Summary of the Risk Management Plan (RMP) for Keytruda. Swissmedic. [Accessed] https://wwwswissmedic.ch/dam/swissmedic/de/dokumente/marktueberwachung/rmp/pembrolizumab_keytruda_ rmp-summary.pdf.download.pdf/Pembrolizumab_KeytrudaSwissRMPsummary-vl.pdf.

28. EAU Guidelines 2021. European Association of Urology. [Accessed August 2021]. Available from: https://uroweb.org/ guidelines/compilations-of-all-guidelines.

29. Powles $\mathrm{T}$ et al. Clinical outcomes in post-operative ctDNA-positive muscle-invasive urothelial carcinom (MIUC) patients ESMO In mus Congress; 9-12 December 2020. Oral presentation 10. 30. Bajorin DF, Witjes JA, Gschwend J, et al. First results from
the phase 3 CheckMate 274 trial of adjuvant nivolumab vs plathe phase 3 CheckMate 274 trial of adjuvant nivolumab vs pla-
cebo in patients who underwent radical surgery for high-risk muscle-invasive urothelial carcinoma (MIUC). J Clin Oncol. 2021;39(6_suppl):391-391. doi:10.1200/JCO.2021.39.6_ suppl.391

31. Apolo AB, Rosenberg JE, Kim WY, et al. Alliance A031501: Phase III randomized adjuvant study of MK-3475 (pembrolizumab) in muscle-invasive and locally advanced urothelial carcinoma (MIBC) (AMBASSADOR) versus observation. J Clin Oncol. 2019;37(7_suppl):TPS504-TPS504. doi:10.1200/JCO.2019.37.7 suppl.TPS504

32. Powles T, Kockx M, Rodriguez-Vida A, et al Clinical ef ficacy and biomarker analysis of neoadjuvant atezolizumab in operable urothelial carcinoma in the ABACUS trial. Nat Med. operable urothelial carcinoma in the ABACUS trial. Nat Med. 33. Necchi A, Anichini A, Raggi D, et al. Pembrolizumab a Neoadjuvant Therapy Before Radical Cystectomy in Patient With Muscle-Invasive Urothelial Bladder Carcinoma (PURE01): An Open-Label, Single-Arm, Phase II Study.J Clin Oncol. 2018;36(34):3353-3360. doi:10.1200/JCO.18.01148

34. Gupta S, Sonpavde G, Weight CJ, et al. Results from BLASST-1 (Bladder Cancer Signal Seeking Trial) of nivolumab, gemcitabine, and cisplatin in muscle invasive bladder cancer (MIBC) undergoing cystectomy. J Clin Oncol. 2020;38(6_suppl):439-439. doi:10.1200/JCO.2020.38.6 suppl.439

35. Pfister C, Gravis G, Flechon A et al. Dose-dense methotrexate, vinblastine, doxorubicin and cisplatin (dd-MVAC) or gemcitabine and cisplatin (GC) as perioperative chemotherapy for patients with muscle-invasive bladder cancer (MIBC) Results of the GETUG/AFU VESPER V05 phase IUI trial. Oral presentation presented at: ESMO Congress 2021; 16-21 September 2021. 